\title{
Deliberate paradigm shift in research in rare neurodevelopmental disorders
}

\author{
Jennifer M. Bain ${ }^{1 *}\left(\mathbb{D}\right.$, Adel Ardalan² and Sylvie Goldman ${ }^{1,3}$
}

\begin{abstract}
Diagnosis and management of children with rare neurodevelopmental disorders (RNDDs) are complex. The COVID19 pandemic has forced us to rethink the research activities critical to improve our understanding and treatment of RNDDs, such as creating large international registries and developing natural history studies. In this communication, we reflect on our latest effort in conducting research remotely while providing support, education and feedback to families affected by a specific RNDD. Specifically, we advocate for a deliberate paradigm shift towards virtual family meetings as ecological platforms to enroll and assess individuals with rare disorders. Herein, we demonstrate that such a shift is crucial to substantially increasing geographical and age range coverage, which are essential for capturing the phenotypic variations in RNDDs. Finally, we call on the community to invest in building integrated technological platforms necessary for effective remote research activities, through standardization, collaboration and training.
\end{abstract}

\section{Introduction}

A rare neurodevelopmental disorder (RNDD) is defined as a neurodevelopmental condition that affects fewer than 200,000 people. The diagnosis and management of children with RNDDs are complex for a variety of reasons, including limited information about these ultra-rare conditions and the prevalence of challenging behaviors. In addition, individuals with RNDDs and their families are often more susceptible to elevated anxiety and social seclusion. These challenges, in turn, make enrollment and research on RNDDs difficult to conduct and sustain.

As a result, comprehensive research programs are essential to refine methods of screening and diagnosis, and to develop targeted treatments for clinical trials. The natural history of many RNDDs needs to be described in detail before an intervention can be proposed that will impact the natural trajectory of such disorders. This calls for large international registries and uninterrupted

\footnotetext{
*Correspondence: jb3634@cumc.columbia.edu

${ }^{1}$ Department of Neurology, Division of Child Neurology, Columbia

University Irving Medical Center, 180 Fort Washington Avenue, 5th Floor, New York, NY 10032, USA

Full list of author information is available at the end of the article
}

natural history studies (NHS). Developing an encompassing natural history requires identification and enrollment of many individuals and their families, often necessitating an international quorum to increase the cohort size. Such enrollment is often challenging due to high cost for participation, language barriers, and time and traveling constraints. These logistical barriers can be further exacerbated by the behavioral challenges of patients with RNDDs.

The multitude of restrictions imposed by COVID-19 pandemic have significantly hindered the NHS of RNDDs and as a result, many research teams (as well as providers and educators) have rushed in to temporarily remedy the situation by moving their activities online, with many of them planning to go back to pre-pandemic practices as soon as the pandemic would be under control. Here, we argue that the RNDD research community should embrace a deliberate and concerted paradigm shift toward sustained online research activities for the following reasons: (1) Virtual family meetings could provide a more accessible, convenient and intimate way for individuals with rare disorders and their families, to participate in research studies; (2) These virtual meetings use more ecological platforms for assessment and treatment original author(s) and the source, provide a link to the Creative Commons licence, and indicate if changes were made. The images or other third party material in this article are included in the article's Creative Commons licence, unless indicated otherwise in a credit line to the material. If material is not included in the article's Creative Commons licence and your intended use is not permitted by statutory regulation or exceeds the permitted use, you will need to obtain permission directly from the copyright holder. To view a copy of this licence, visit http://creativecommons.org/licenses/by/4.0/. The Creative Commons Public Domain Dedication waiver (http://creativeco mmons.org/publicdomain/zero/1.0/) applies to the data made available in this article, unless otherwise stated in a credit line to the data. 
of individuals with RNDDs; and (3) Remote enrollment can substantially increase the age range and geographical coverage of the studies, which are vital for capturing the phenotypic spectrum of RNDDs.

Here, we report on our most recent effort in conducting research remotely to provide support, education and feedback to families affected by a specific RNDD and share the lessons we learned from our experience. Due to pandemic-related restrictions our annual in-person family meeting could not be conducted as usually planned; no in-person data could be collected, and no social gathering could be organized around lectures and lunches. However, as the continuity of care and research was paramount, we decided to shift our family meeting to a virtual format to avoid a disruption in our natural history study and other research efforts. Our study was unique in its depth (detailed behavioral and computational studies), breadth (natural history, physical and occupational therapy, gait analysis) and scale (geographical coverage and age range).

\section{Study outline}

Our team has focused on understanding the developmental trajectory of individuals with $H N R N P H 2$ gene variants associated with a neurodevelopmental disorder [1, 2]. HNRNPH2-related disorder (OMIM 300,986 Mental retardation, X-linked, syndromic, Bain type; MRXSB) is a newly described neurodevelopmental disorder caused by pathogenic variants in the HNRNPH 2 gene $[1,2]$. HNRNPH2 is located on the $\mathrm{X}$ chromosome and encodes Heterogeneous Nuclear Ribonucleoprotein H2 (HNRNPH2), a ubiquitously expressed RNA-binding protein that primarily functions to regulate the alternative splicing of pre-mRNA [3, 4]. HNRNPH2 is one of several HNRNPs that are associated with a neurodevelopmental disorder $[5,6]$. Bain et al. initially described HNRNPH2-related disorder in 6 females with a similar neurodevelopmental phenotype including global developmental delay/ intellectual disability, severe motor impairment, musculoskeletal problems and neuropsychiatric diagnoses [5]. We recently expanded on the phenotype including 33 individuals and a more detailed clinical description of the disorder [6].

Since the initial description in 2016, we have aligned with the parent-run non-profit organization Yellow Brick Road Project (YBRP) to increase awareness about the importance of participating in a natural history study (Fig. 1a). Within a year of initial publication, we started a natural history study, and we continue to enroll new subjects monthly (www.hnrnph2.com). Participants were referred to this study (ClinicalTrials.gov NCT03492060) if they were diagnosed with pathogenic or likely pathogenic variants in Heterogeneous Nuclear
Ribonucleoprotein H2 (HNRNPH2) using ACMG/AMP criteria, largely identified via clinical exome sequencing. The study was approved by the ethic committee of the Columbia University Institutional Review Board and informed consent was obtained from all caregivers or legal guardians. Our research protocol and data results is available upon request to the authors for our Natural History Study and Virtual Shift Protocol.

In close collaboration with YBRP, we held annual family meetings in 2018 and 2019, during which time our enrollment increased (Fig. 1a). In-person assessments included neurological, medical and orthopedic examinations as well as physical and occupational therapy evaluations. At the last in-person meeting, in New York City which was at a local conference center with guest rooms and leisure spaces like a gym and pool, families were able to schedule their research evaluations as well as plan for rest time and breaks when needed. There was also a dedicated space for care and play time provided for affected individuals to be cared for while parents and families participated in lectures and meetings with other research activities.

Given the significant motor impairments in this group, characterizing the motor delays and dysfunction has been a major focus of our research team and we sought to capture data from various sources. At the start of each annual meeting, each family completed the Pediatric Evaluation of Disability Inventory Computer Adaptive Test (PEDI-CAT), which is a caregiver-reported outcome measure that evaluates functional domains including daily activities, mobility, social/cognitive and responsibility [7-9]. The instrument was designed to evaluate individuals ages birth to 21 years old and has been validated for children with disabilities over a variety of diagnoses including autism spectrum disorder, Rett Syndrome and spinal muscular atrophy $[10,11]$. After completion of the PEDI-CAT, each participant was evaluated by a trained physical therapist to measure motor skills using the gross motor function measure (GMFM-88). The GMFM-88 is an in person 88 -item evaluative tool that was initially designed and validated to measure gross motor function in children with cerebral palsy [12]. The GMFM-88 assesses a wide spectrum of gross motor activities including the following five dimensions: (A) lying and rolling, (B) sitting, (C) crawling and kneeling, (D) standing, and (E) walking, running, and jumping. Each item is scored on a 4-point scale with a maximum total raw score of 256 points and can be used into adulthood. Items from the GMFM- 88 have been used to develop scales to measure motor function in Down Syndrome [13], spinal muscular atrophy [14] and even neurodevelopmental disorders such as Rett Syndrome [15]. We have previously shown strong correlation with the parent-report PEDI-CAT 


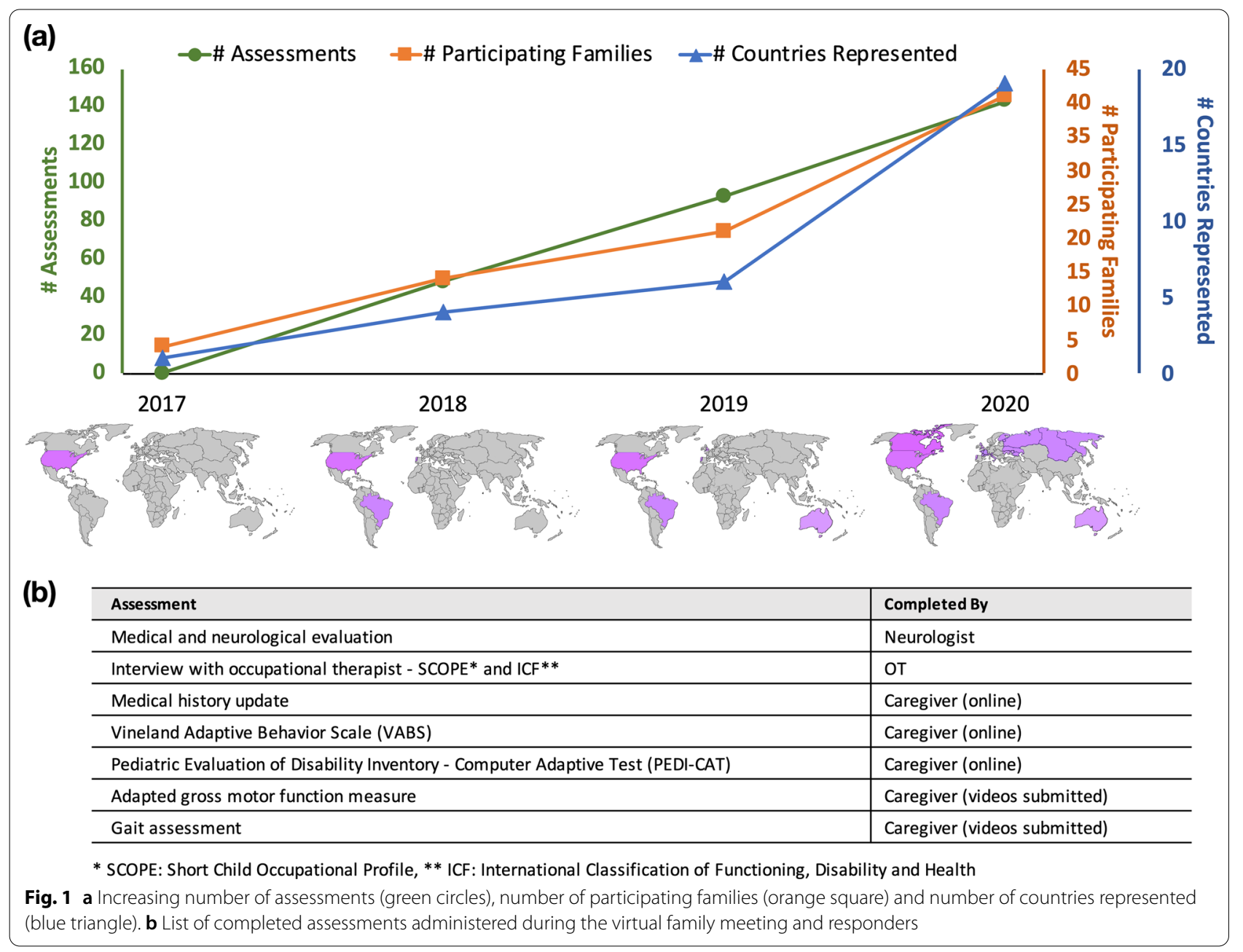

and clinician-administered GMFM (publication under review). Lastly, given that gait difficulties were identified as an intrusive symptom in this disorder by families, gait analysis was studied using insole technology and gait mat (Fig. 2a) [16]. The protocol of our natural history focused on standardized neurological examination and physical and occupational therapists' evaluation. Further, cognitive level was assessed using the Vineland Adaptive Behaviors Scale rather than formal neurocognitive testing which may represent a limitation.

During this global pandemic, we sought to carry on with our NHS, with concern that the loss of a single year worth of data and interactions with the families would be detrimental to the progress of the study. In lieu of our regular in-person meeting, we worked closely with YBRP to shift to a virtual meeting, including one-on-one interviews, virtual video and questionnaire data collection and online family gatherings (Fig. 1b). In addition to data collection, the family meeting itself included 3 days of virtual conferences, roundtable discussions and social gatherings. Prior to the meeting, we provided a detailed illustrated instruction manual for scheduling video visits with the child neurologist and occupational therapy team, as well as how to collect and send behavioral data and fill out questionnaires. For the gross motor physical therapy assessment, we chose 23 items from the GMFM- 88 instrument for individuals to perform at home which covered the main gross motor domains and captured both floor and ceiling effects of the participants' motor profile. Families were instructed to videotape the motor tasks and upload them via online databanks. We offered individual physical therapy sessions for those families who needed further instructions or support to complete the assessment. In lieu of using in-sole shoe sensors with gait mat (Fig. 2a), we asked families to videotape specific gait tasks using our illustrated instruction checklist for later artificial intelligence (AI)-based pose estimation [17]. Figure 2b shows an example from 
(a)

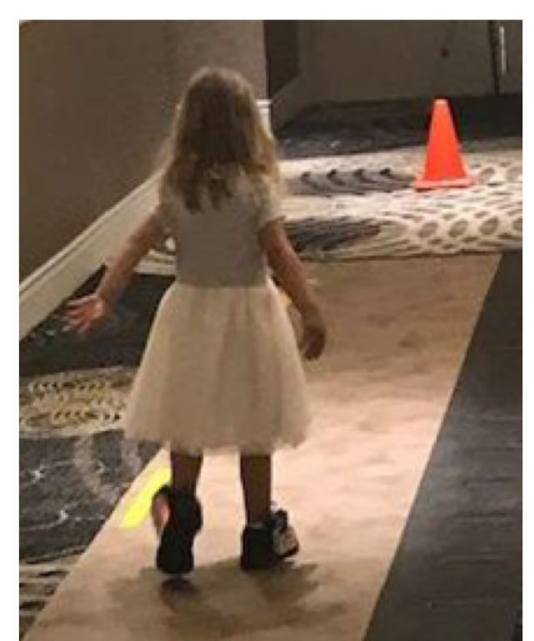

(b)

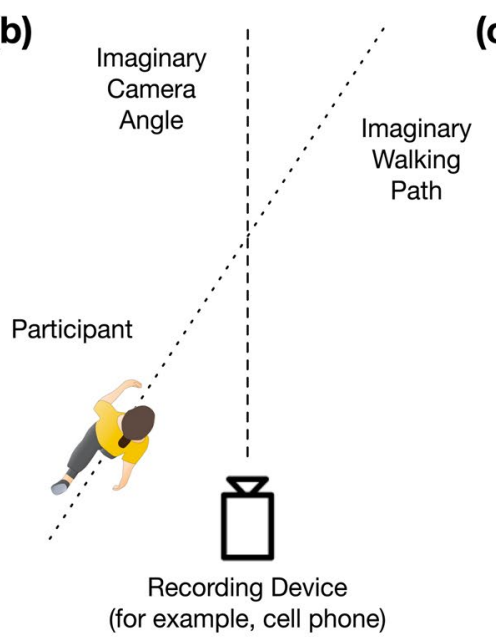

(c)

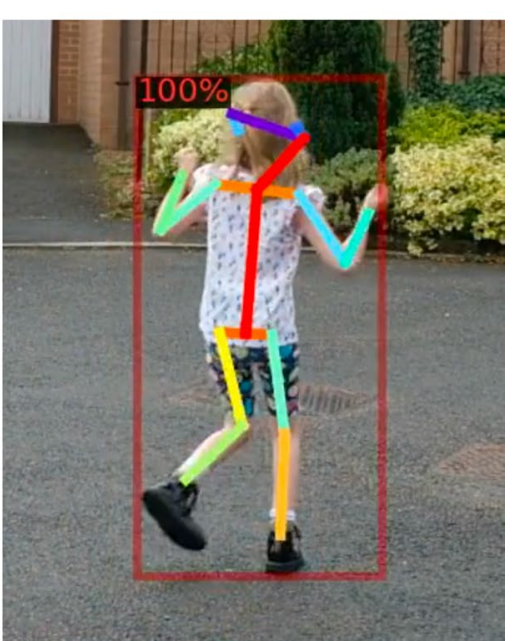

Fig. 2 a Toddler girl walking on a sensor-walkway mat wearing instrumented sneakers. b Instructions for gait videos recording. c Al based pose estimation extracted from home videos

the gait data collection instructions and Fig. 2c shows a sample result of extracting gait information using AI.

In addition to the gross motor and gait evaluations, we sought to further investigate fine motor deficits and sensory issues. This year's assessment included a qualitative videotaped interview to complete the Short Childhood Occupational Profile (SCOPE) [18]. The OT team then combined the data from this interview with their completion of other standardized measures such as the Vineland Adaptive Behavior Scale 3rd Edition and Behavior Assessment System for Children $3^{\text {rd }}$ Edition to assign each subject an ICF score [19, 20]. The International Classification of Functioning, Disability and Health, known more commonly as ICF, is a classification of health and health-related domains [21]. This evaluation will be utilized for a deeper understanding of the occupational therapist needs by the group, as well as providing feedback to the family for further clinical recommendations.

At the conclusion of the family meeting, we held a virtual focus group to gather families' feedback on the process, as well as to reinforce the sense of community and continuity of care between the research team and family organization. We then distributed a survey to evaluate families' satisfaction, identify specific issues and to inform future meetings (Fig. 3). Most of the feedback was positive regarding the experience of participants in a virtual meeting and many endorsed a positive response about future engagement with a virtual setting.

\section{Obstacles and disadvantages}

One limitation of performing the natural history study in the virtual paradigm was the limited validity in telehealth cognitive testing. Since the start of the pandemic the lack of validity of remote cognitive assessment in children is a central debate in the field of neuropsychology. In order to mitigate this issue, we have followed the recommendations of the profession and administered the Vineland Adaptive Behavior Scale-Parents Interview to document cognitive changes for this year $[22,23]$.

Another limitation of this virtual platform was the loss of intimate connection in meeting families, clinicians and researchers during an in-person event. As noted in some of the qualitative feedback from families, these annual meetings often brought a deep feeling of belonging and an opportunity to learn from experts and other families. In the end, however, most families did report an overall positive experience and a strong desire to move forward and participate in future meetings as their way to contribute to scientific progress for their own child and the community of RNDD.

In regard to the technical setup, one obstacle in data collection was the challenge to obtain videos suitable for offline gait analysis. The current state of the art in pose estimation from video recordings remains sensitive to various parameters such as angle, lighting, occlusions, etc. Although we collected high-quality videos and were able to generate pose estimation data by providing detailed instructions and feedback to the caregivers on their initial recordings, they often had to repeat the recording to meet our criteria. With the advances in pose estimation technology, we expect that future data collection will be easier and more efficient. We also plan on using parents' feedback to revise our instructions to make them clearer for higher quality standard.

Lastly, while most families reported small technical difficulties using the virtual platform, we are aware that 


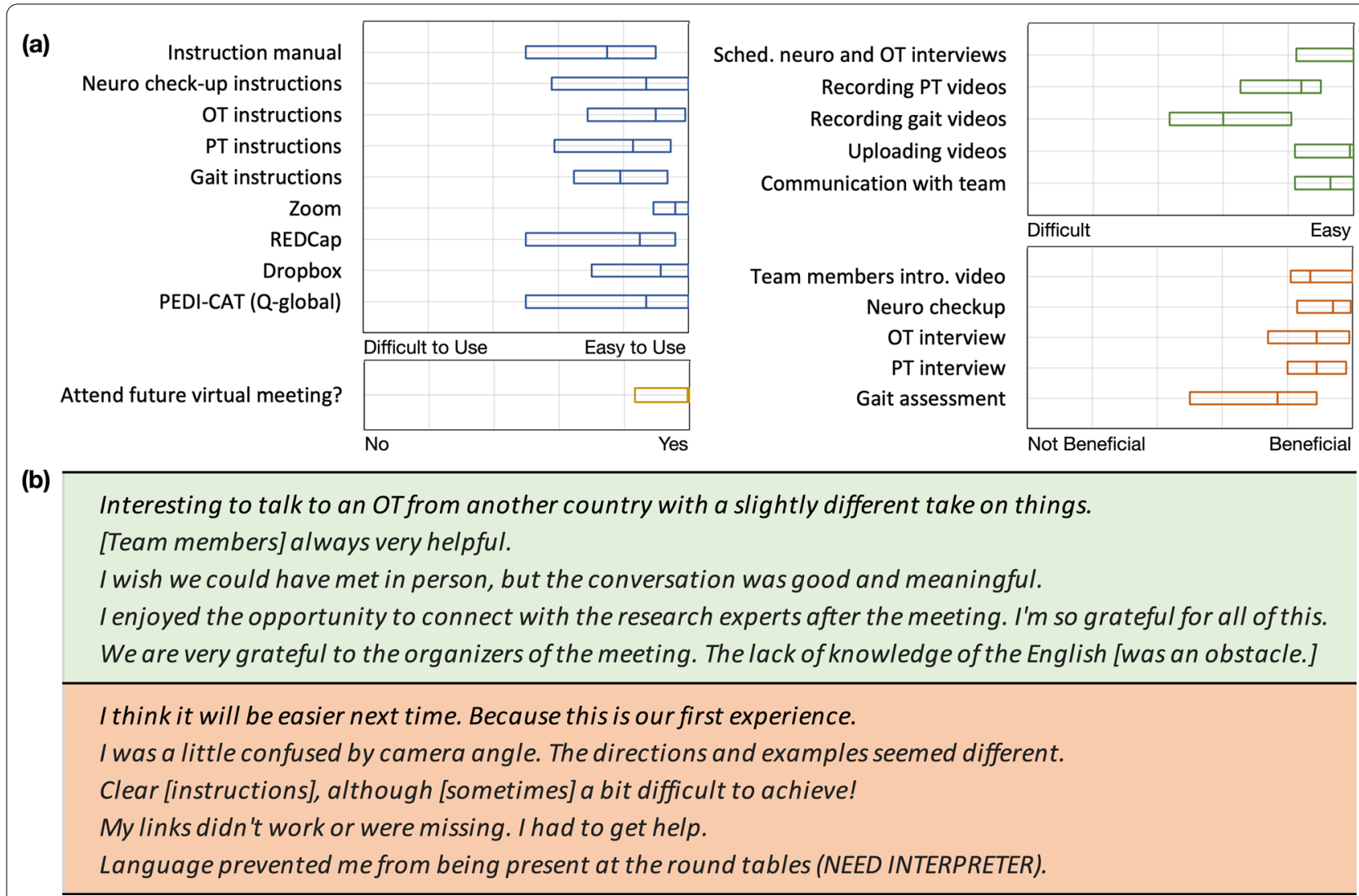

Fig. 3 a Summary of the survey results by question type. $\mathbf{b}$ Examples of positive feedback (green) and issues (orange) raised by participating families

several families were unable to participate due to limited access to video collection methods, computer systems or high-speed internet connections. Functional level of families to be able to read and follow the instructions provided to families in the instruction manual and use the technology needed for virtual meetings. There were also other families who are unable to provide care to their family member with special needs while also participating in the meeting itself. We also recognize the literacy and language barriers, as well as difficulties for a subset of families to be available for full days of meeting while caring for their child or young adults with special needs.

\section{Lessons learned}

Based on the results from the focus group and the survey, we are highlighting the following points which we think will be useful for the RNDD research community to continue to provide high-quality care and study of individuals and families with RNDD while adapting to the new conditions.
- Virtual family meetings provide an ecological and intimate platform for valid assessment and treatment of individuals with rare disorders.

- Patients and their caregivers appeared comfortable and not pressured while performing the tasks in their homes.

- Although the families expressed grief for the lost sense of community and care they felt during in-person family meetings, they were happy to get a chance to contribute to the study, check in with researchers, practitioners and other families during these hard times, as well as receive information and feedback about their children's progress.

Thanks to this paradigm shift, we were able to substantially increase the geographical coverage of our meeting. As shown in Fig. 1a, the number of international families who participated in the meeting tripled compared to the previous year. This was mostly due to reduced cost of participation, easier access, and wider reach of the recruitment effort. Moreover, we were able to extend the overall data collection time and thus avoid schedule 
conflict with other conferences or responsibilities within this short time period.

A wider geographical coverage is vital within RNDDs in order to include wider phenotypic variations, extend enrollment and care to under-privileged families as well as those reluctant to travel with their family member. While we were able to include a wider geographic area and collect more phenotypic variability, we were unable to demonstrate engagement with more under-privileged families. Future endeavors should seek to demonstrate the effective reach of such studies to individuals and families with currently limited access to high-speed internet and video capability. We are hopeful that the emergence of new internet technologies (e.g. recent World Mobile Group projects in Tanzania) and low-cost cameras (e.g. on affordable cell phones) would enable more underprivileged families to join future studies. Altogether, this experience helped us and the scientific community to practice how to build stronger and more global links with families.

We recognize that the integration of new technological tools requires effort to increase standardization, unification and training. Indeed, the research team and the participating families mentioned a lack of standardized streamlined technological platforms to encompass all planning, training, data collection and analysis that would facilitate a unified framework. The use of a series of tools for each assessment made participation more difficult and time-consuming for families.

In conclusion, in the era of precision medicine for the treatment of rare disorders, it is imperative and feasible to shift the research paradigm and continue gathering critical data. While a complete shift to virtual platform may still be complex, we suggest there is a need to incorporate data collected from this medium to complement in-person evaluations. We argue that this approach is essential to thrust robust and rigorous clinical trials and support families in dire need during these stressful times.

Resources available for families affected by rare diseases in the setting of the COVID19 pandemic and for researchers include:

- https://autismsciencefoundation.org/covid-19-resou rces/

- https://rarediseases.org/covid-19/

- https://globalgenes.org/2020/03/20/supporting-therare-disease-communityduring-covid-19/

- https://www.rarechromo.org/covid19update/

\section{Abbreviations}

NHS: Natural history study; GMFM: Gross motor function measure; HNRNPH2: Heterogeneous Nuclear Ribonucleoprotein H2; ICF: International Classification of Functioning, Disability and Health; PEDI-CAT: Pediatric Evaluation of Disability Inventory Computer Adaptive Test; RNDD: Rare neurodevelopmental disorders; YBRP: Yellow Brick Road Project; SCOPE: Short Childhood Occupational Profile.

\section{Acknowledgements}

We gratefully acknowledge all of the families from the Yellow Brick Road Project who support this Natural History Study, as well as data provided by Simons Searchlight and SFARI sponsorship. AA wants to thank Amir H. Assad for the invaluable discussion which significantly improved the quality of the present work. Thank you for our collaborative virtual study team, notably our physical and occupational therapists and research coordinator Olivia Thornburg for making a virtual family meeting vision a reality.

\section{Authors' contributions}

All three authors contributed equally to the design, coordination, drafting and revising of this manuscript. All authors read and approved the final manuscript.

\section{Funding}

There is no funding source for the data included in this manuscript.

Availability of data and materials

We will provide our protocol upon request to the authors.

\section{Declarations}

Ethical approval and consent to participate

This study was approved by Columbia University Institutional Review Board and each participant has consented to publication.

\section{Consent for publication}

The study team has obtained informed consent from participants in which the process notes that surveys such as included in this manuscript include the right to publish the results. Moreover, the figure contains a picture of a subject from our study, and we have consent for use of data including photos for publication. A compilation of our entire informed consents for those in our study can be submitted upon request from the Editor.

\section{Competing Interests}

There are no competing interests relevant to this study.

\section{Author details}

${ }^{1}$ Department of Neurology, Division of Child Neurology, Columbia University Irving Medical Center, 180 Fort Washington Avenue, 5th Floor, New York, NY 10032, USA. ${ }^{2}$ Zuckerman Mind Brain Behavior Institute, Columbia University, 3227 Broadway, New York, NY 10027, USA. ${ }^{3}$ Department of Neurology, Division of Cognitive Neuroscience, Columbia University Irving Medical Center, 622 West 168th Street, PH18-331, New York, NY 10032, USA.

Received: 2 December 2020 Accepted: 21 May 2021

Published online: 09 June 2021

\section{References}

1. Bain JM, Cho MT, Telegrafi A, et al. Variants in hnrnph2 on the x chromosome are associated with a neurodevelopmental disorder in females. Am J Human Genet. 2016;99(3):728-34. https://doi.org/10.1016/j.ajhg.2016. 06.028 . 
2. Bain JM, et al. Detailed clinical and psychological phenotype of the X-linked HNRNPH2-related neurodevelopmental disorder. Neurol Genet. 2021;7(1):551. https://doi.org/10.1212/NXG.0000000000000551.

3. Feng $D$, Xie J. Aberrant splicing in neurological diseases. Wiley Interdiscip Rev RNA. 2013;4:631-49.

4. Linder B, Fischer U, Gehring NH. mRNA metabolism and neuronal disease. FEBS Lett. 2015;589:1598-606.

5. Wang T, Hoekzema K, Vecchio D, Wu H, Sulovari A, Coe BP, Gillentine MA, Wilfert AB, Perez-Jurado LA, Kvarnung M, Sleyp Y, Earl RK, Rosenfeld JA Geisheker MR, Han L, Du B, Barnett C, Thompson E, Shaw M, Carroll R, Friend K, Catford R, Palmer EE, Zou X, Ou J, Li H, Guo H, Gerdts J, Avola E, Calabrese G, Elia M, Greco D, Lindstrand A, Nordgren A, Anderlid BM, Vandeweyer G, Van Dijck A, Van der Aa N, McKenna B, Hancarova M, Bendova S, Havlovicova M, Malerba G, Bernardina BD, Muglia P, van Haeringen A, Hoffer MJV, Franke B, Cappuccio G, Delatycki M, Lockhart PJ, Manning MA, Liu P, Scheffer IE, Brunetti-Pierri N, Rommelse N, Amaral DG, Santen GWE, Trabetti E, Sedláček Z, Michaelson JJ, Pierce K, Courchesne E, Kooy RF; SPARK Consortium, Nordenskjöld M, Romano C, Peeters H, Bernier RA, Gecz J, Xia K, Eichler EE. Large-scale targeted sequencing identifies risk genes for neurodevelopmental disorders. Nat Commun. 2020 Oct 1;11(1):4932. https://doi.org/10.1038/s41467-020-18723-y. Erratum in: Nat Commun. 2020 Oct 21;11(1):5398.

6. Low YH, Asi Y, Foti SC, Lashley T. Heterogeneous nuclear ribonucleoproteins: implications in neurological diseases. Mol Neurobiol. 2021 Feb;58(2):631-46. https://doi.org/10.1007/s12035-020-02137-4.

7. Dumas HM, et al. Construct validity of the pediatric evaluation of disability inventory computer adaptive test (PEDI-CAT) in children with medical complexity. Disabil Rehabil. 2017;39(23):2446-51

8. Dumas HM, Fragala-Pinkham MA. Concurrent validity and reliability of the pediatric evaluation of disability inventory-computer adaptive test mobility domain. Pediatr Phys Ther. 2012;24(2):171-6.

9. Haley SM, et al. Accuracy and precision of the Pediatric Evaluation of Disability Inventory computer-adaptive tests (PEDI-CAT). Dev Med Child Neurol. 2011;53(12):1100-6.

10. Kao YC, et al. Comparing the functional performance of children and youths with autism, developmental disabilities, and no disability using the revised pediatric evaluation of disability inventory item banks. Am J Occup Ther. 2012;66(5):607-16.

11. Kramer JM, Liljenquist $K$, Coster WJ. Validity, reliability, and usability of the pediatric evaluation of disability inventory-computer adaptive test for autism spectrum disorders. Dev Med Child Neurol. 2016;58(3):255-61.

12. Russell DJ, Rosenbaum PL, Cadman DT, Gowland C, Hardy S, Jarvis S. The gross motor function measure: a means to evaluate the effects of physical therapy. Dev Med Child Neurol. 1989 Jun;31(3):341-52. https:// doi.org/10.1111/j.1469-8749.1989.tb04003.x.

13. Russell D, Palisano R, Walter S, Rosenbaum P, Gemus M, Gowland C, Galuppi B, Lane M. Evaluating motor function in children with Down syndrome: validity of the GMFM. Dev Med Child Neurol. 1998 Oct;40(10):693-701

14. Nelson L, Owens H, Hynan LS, lannaccone ST, Group A. The gross motor function measureTM is a valid and sensitive outcome measure for spinal muscular atrophy. Neuromuscul Disord. 2006 Jun 1;16(6):374-80.

15. Downs J, Stahlhut M, Wong K, et al. Validating the Rett Syndrome Gross Motor Scale. PLoS ONE. 2016;11:e0147555.

16. Duong TTH, Goldman S, Zhang H, et al. Validation of Insole-based Gait Analysis System in Young Children with a Neurodevelopmental Disorder and Autism Traits. In: 2020 8th IEEE RAS/EMBS International Conference for Biomedical Robotics and Biomechatronics (BioRob). ; 2020:715-720. doi:https://doi.org/10.1109/BioRob49111.2020.9224273

17. Wu Y, Kirillov A, Massa F, Lo W, Girshick R. Detectron2. Accessed October 16, 2020. https://github.com/facebookresearch/detectron2

18. Bowyer PL, Kramer J, Kielhofner G, Maziero-Barbosa V, Girolami G. Measurement properties of the Short Child Occupational Profile (SCOPE). Phys Occup Ther Pediatr. 2007;27(4):67-85.

19. Reynolds CR, Kamphaus RW. Behavior Assessment System for Children. Third edition (BASC 3). Bloomington, MN: Pearson. 2015.

20. Sparrow SS, Cicchetti DV, Saulnier CA. Vineland adaptive behavior scales: Third edition (Vineland-3). Bloomington: NCS Pearson; 2016.

21. World Health Organization. The international classification of functioning, disability and health (ICF). Geneva: WHO; 2001.

22. Dahiya AV, DeLucia E, McDonnell CG, Scarpa A. A systematic review of technological approaches for autism spectrum disorder assessment in children: Implications for the COVID-19 pandemic. Res Dev Disabil. 2021 Feb:109:103852. https://doi.org/10.1016/j.ridd.2021.103852.

23. Berger NI, Wainer AL, Kuhn J, et al. Characterizing available tools for synchronous virtual assessment of toddlers with suspected autism spectrum disorder: a brief report. J Autism Dev Disord. 2021. https://doi.org/10. 1007/s10803-021-04911-2.

\section{Publisher's Note}

Springer Nature remains neutral with regard to jurisdictional claims in published maps and institutional affiliations.
Ready to submit your research? Choose BMC and benefit from:

- fast, convenient online submission

- thorough peer review by experienced researchers in your field

- rapid publication on acceptance

- support for research data, including large and complex data types

- gold Open Access which fosters wider collaboration and increased citations

- maximum visibility for your research: over 100M website views per year

At BMC, research is always in progress.

Learn more biomedcentral.com/submissions 